\title{
Influence du type de nutrition azotée sur le déclenchement de la chlorose du sapin de Nordmann (Abies nordmanniana, Spach, 1842)
}

\author{
N. Khalil1* ${ }^{\star}$, C. Leyval ${ }^{1}$, M. Bonneau² et B. Guillet ${ }^{1}$ \\ 1 Centre de pédologie biologique (LP 6831 du CNRS, associé à l'université de Nancy I), BP 5, \\ 54501 Vandœuvre-lès-Nancy Cedex; \\ 2 CNRF, INRA, Champenoux, 54280 Seichamps, France;
}

(reçu le ter avril 1988; accepté le 10 mars 1989)

Résumé - Des cas de chlorose ont été signalés plusieurs fois chez le sapin de Nordmann cultivé sur des sols carbonatés. Les symptômes apparaissent dès le jeune âge et se manifestent par un jaunissement des aiguilles. Parmi les facteurs qui sont censés déclencher la chlorose, le calcaire des sols et les ions carbonate-bicarbonate des solutions des sols, la structure et la texture des sols et enfin le type de nutrition azotée sont souvent présentés comme des causes agissantes.

Dans une recherche précédente (Khalil et al., 1988) concernant la chlorose du sapin de Nordmann en milieu naturel, il avait été montré que, parmi les facteurs du sol, le $\mathrm{pH}$ et la teneur en calcaire total rendent le mieux compte des symptomes chlorotiques. Mais pour tenter de discerner l'action du calcium de celle des ions bicarbonate-carbonate et apprécier les effets de la nutrition en azote, une série d'expériences de croissance de plants fut réalisée en faisant varier les facteurs suivants : le calcium, les ions $\mathrm{CO}_{3}^{2-} / \mathrm{HCO}_{3}^{-}$et le type d'alimentation azotée.

Les cultures ont été faites en serre sur support inerte quartzeux (sable de Fontainebleau) qui a été enrichi ou non, soit en carbonate de calcium (craie) pour juger de l'influence du $\mathrm{Ca}^{2+}$ et des ions $\mathrm{CO}_{3}^{2-} / \mathrm{HCO}_{3}^{-}$, soit en sulfate de calcium (gypse) pour apprécier le rôle des ions $\mathrm{Ca}^{2+}$ seuls; 12 traitements (Tableau I) ont été mis en place. Les supports placés dans des tubes ont reçu une plantule de 2 ans. Les plants ont été cultivés du 11 décembre 1985 au 22 octobre 1986 et arrosés (10 $\mathrm{ml} / \mathrm{sem}$ ) par des solutions nutritives de deux sortes ne différant que par la forme sous laquelle l'azote était fourni, soit nitrique, soit ammoniacale (Tableau II). Les percolats ont été recueillis, regroupés mensuellement et analysés. Après observation de l'état des plants en fin de culture, les aiguilles jeunes sont collectées, séchées et pesées ainsi que les racines, préalablement lavées. L'état des plants en fin de culture (Tableau IV) permet de conclure à l'existence de deux types de chlorose chez le sapin de Nordmann. En condition d'alimentation ammoniacale, la chlorose se manifeste uniquement en présence de $\mathrm{CaCO}_{3}$. La chlorose est bien plus sévère en condition d'alimentation nitrique, car même les plants des témoins sont affectés.

En alimentation ammoniacale, la chlorose se développe uniquement en présence de $\mathrm{CaCO}_{3}$. En présence de gypse, il y a seulement dépression de la croissance comme le prouve la comparaison avec le témoin des biomasses foliaires et racinaires (Figs. 1, 2A et B). En environnement calcaire

*Actuellement : université de Tichrine, Lattaquié, Syrie. 
caractérisé par le pH élevé et la forte alcalinité (Tableau III) des solutions percolantes, la chlorose se définit par les points suivants :

- diminution de la teneur des aiguilles en fer extractible par $\mathrm{HCl}$, en magnésium et en soufre (Fig. 3),

- accroissement du calcium des aiguilles (Fig. 3) et surtout des racines (Fig. 4),

- alimentation difficile en potassium et surtout réduction de la mobilité de l'élément entre les racines et les aiguilles car les différences de teneur selon les traitements sont bien plus fortes dans les aiguilles.

Dans tous les cas, la nutrition azotée demeure normale.

En alimentation nitrique, la chlorose se développe, même en absence de $\mathrm{CaCO}_{3}$, et n'est pas sensiblement augmentée en présence de calcaire. La chlorose "nitrique" se caractérise essentiellement par une très forte carence en azote total révélée aussi bien par l'analyse des aiguilles (Fig. 3) que des racines (Fig. 4). Le sapin de Nordmann se comporte comne une espéce "nitratophobe» qui présenterait des difficultés à réduire l'azote nitrique absorbé. La chlorose se caractérise au niveau des aiguilles par un déficit des teneurs en fer soluble dans $\mathrm{HCl}$ et en magnésium, si l'on prend comme référence le témoin à alimentation ammoniacale.

Les deux types de chlorose ont en commun le fait d'avoir bloqué au niveau de leurs racines une importante quantité de calcium qui forme un manchon de carbonate résultant de la précipitation à partir de solutions riches en bicarbonates.

\begin{abstract}
Abies nordmanniana - chlorose calcaire - nutrition azotée - nutrition minérale - sol calcaire
\end{abstract}

Summary - Influence of the type of nitrogen nutrition on the onset of chlorotic symptoms in the Nordmann fir (Ables nordmanniana, Spach, 1842). Cases of chlorosis have been frequently mentioned in the Nordmann fir (Abies nordmanniana) growing on calcareous soils. Symptoms appear during the early stages of growth and are diagnosed by a needle yellowing. Among the factors implicated in triggering chlorosis, the soil carbonates, bicarbonate-carbonate ionic activity of the soil solution, structure and texture of the soils and the kind of nitrogen nutrition are frequently cited.

The characteristics of chlorosis have been studied in the field and have been presented and discussed in another paper (Khalil et al., 1988). Healthy green plants and other yellowing smaller ones were sampled in a young plantation established on soil developed on friable calcareous marl near Carcassonne (Eastern Pyrénées, South France). The $\mathrm{pH}$ and the total carbonate content are the soil factors which are the most relevant to the chlorosis symptoms. Regarding needle analyses, the chlorosis symptoms were found to be correlated with a decrease in $\mathrm{Fe}, \mathrm{Mg}, \mathrm{Mn}, \mathrm{N}$ and $\mathrm{S}$, the essential elements for chlorophyl synthesis. A plant culture experiment carried out in the greenhouse with a culture support enriched with $\mathrm{CaCO}_{3}$ and a high packirg density demonstrated that $\mathrm{CaCO}_{3}$ and a decrease in porosity have a positive effect on onset of yellowing.

To try to distinguish calcium action from that of bicarbonate-carbonate ions and to evaluate the effect of the nitrogen nutrition type, a set of experiments on plant growth was programmed with the following factors as variables : calcium, $\mathrm{HCO}_{3}^{-} / \mathrm{CO}_{3}^{2-}$ ions, nitrogen supply in the form of ammonium or nitrate.

Cultures were carried out on inert mineral material (quartzic Fontainebleau sand) in a greenhouse. This culture support was enriched or not either with $\mathrm{CaCO}_{3}$ (chalk) to determine the influence of $\mathrm{Ca}^{2+}$ and $\mathrm{HCO}_{3}^{-} / \mathrm{CO}_{3}^{2-}$ ionic couple, or with $\mathrm{CaSO}_{4}$ (gypsum) to evaluate the role of $\mathrm{Ca}^{2+}$ alone. Twelve treatments (Table I) were prepared with or without a $\mathrm{Fe}^{2+}$ supply from the nutrient solution. The mineral culture supports were placed in tubes with a 2-year-old plant. Plants (Abies nordmanniana) were cultivated from the 11th December 1985 to the 22th October 1986 and irrigated (10 $\mathrm{m} /$ week) with two kinds of nutrient solutions differing only in the form of nitrogen supply: ammonium or nitrate. The leaching solutions were collected weekly and bulked and analyzed monthly. After observations at the end of the experiment, the young needles and the previously 
washed roots were collected, dried, weighed and analyzed. At the end of the culture, the state of the plants (Table IV) suggested the existence of two kinds of chlorosis. With ammoniacal nutrition, chlorosis appeared only in the presence of $\mathrm{CaCO}_{3}$, whereas it was more pronounced with nitrate nutrition as even control plants deteriorated.

With ammoniacal nutrition, chlorosis only developed in the presence of $\mathrm{CaCO}_{3}$. When gypsum was present, the plant growth was only depressed, as proved by the decrease in the needle and root biomass in comparison with the plant control (Figs. 1,2A and 2B). The carbonate environment was characterized by high $\mathrm{pH}$ values and alkaline (Table III) leaching solutions. Chlorosis is defined by :

- a decrease in the HCl-extractable Fe content and the $\mathrm{Mg}$ and $\mathrm{S}$ content of the needles (Fig. 3);

- a Ca increase in the needles (Fig. 3) and particularly in the roots (Fig. 4);

- difficulty in potassium nutrition and in particular a strong reduction in the facility or transfer of this element between the roots and needles;

- an apparently normal nitrogen nutrition throughout.

With nitrate nutrition, chlorosis develops even in the absence of $\mathrm{CaCO}_{3}$. "Nitrate" chlorosis is essentially characterized by a very strong deficiency in total nitrogen displayed by the needle analyses (Fig. 3) and also by the root analyses (Fig. 4).

The Nordmann fir behaves as a "nitratophobic" species that has difficulty in reducing the absorbed nitrate nitrogen. At the needle level, chlorosis is characterized by a decrease in the $\mathrm{HCl}$ extractable $\mathrm{Fe}$ and $\mathrm{Mg}$ contents if the plant control cultivated in ammoniacal conditions is considered as reference.

Moreover, the common feature of the two kinds of chlorosis is the accumulation of a large quantity of calcium at the root level. This is due to the formation of calcium carbonate coatings (Figs. 5 and 6) resulting from $\mathrm{CaCO}_{3}$ precipitation from the bicarbonate-rich solutions as shown by the carbon isotope analyses.

Abies Nordmanniana - lime-induced chlorosis - nitrogen nutrition - mineral nutrition calcareous soils

\section{INTRODUCTION GENERALE}

II a souvent été conseillé d'introduire du sapin de Nordmann sur des sols développés sur roches calcaires (Pourtet, 1951; Association Technique pour la Vulgarisation Forestière, 1966) sans qu'on ait fait clairement la différence entre les sols calciques décarbonatés sur la majeure partie du profil et les sols carbonatés contenant du calcaire total et actif jusqu'à la surface. Or, on a pu remarquer à diverses reprises que sur sols carbonatés le sapin de Nordmann présente, au moins dans le jeune âge, des signes évidents de chlorose (Etaix, 1970; Garbaye \& Le Tacon, 1974). Cette maladie physiologique se manifeste sous la forme d'un jaunissement des aiguilles. Ces symptômes apparaissent d'abord sur les aiguilles les plus jeunes, puis progressivement sur les aiguilles les plus âgées.

Lors d'une recherche récente (Khalil, 1987; Khalil et al., 1988) réalisée sur une station située dans la forêt de Greffeil à 16 $\mathrm{km}$ au sud-est de Carcassonne, il est apparu que le $\mathrm{pH}$ des sols et leur teneur en calcaire total étaient les caractéristiques des sols rendant le mieux compte du déclenchement des symptômes chlorotiques. Quant aux différences entre les aiguilles vertes des plants sains et jaunes des plants chlorosés, elles se manifestent plus spécifiquement sur un ou plusieurs éléments, jouant d'une façon ou d'une autre un rôle sur l'élaboration chlorophyl- 
lienne. II s'agit notamment de l'azote, du soufre, du magnésium, du manganèse et du fer extractible à l'acide chlorhydrique. En outre, lors d'une expérience complémentaire (Khali, 1987) faite en serre et consistant à cultiver des jeunes plants de sapin de Nordmann en pots sur un sol brun lessivé enrichi ou non en carbonate de calcium, le sol étant tassé ou non, on a pu montrer que la réduction de la porosité et le maintien d'une humidité constante du sol aggravaient considérablement les symptômes de chlorose calcaire. Au plan nutritionnel, on observait une augmentation de l'absorption du calcium et une réduction de celle du fer et du manganèse par les plantes (Khalil et al., 1988).

Ces expériences conduisent, comme beaucoup d'autres, au constat que le calcaire favorise le déclenchement de la chlorose. II est d'ailleurs bien connu que les ions $\mathrm{CO}_{3}^{2-} / \mathrm{HCO}_{3}^{-}$peuvent jouer un rôle direct ou indirect dans la manifestation de cette maladie (Brown, 1960; Morlat et al., 1980), car ils perturbent dans les racines la synthèse de l'acide citrique dont la présence est indispensable pour assurer le transport du fer vers les parties aériennes (Gay-Bellile, 1972; Vankat-Rajin et al. 1972; Loué, 1983).

Toutefois, une question reste posée. II s'agit en effet de reconnaître qui, du calcium ou des anions bicarbonates-carbonates, est agent actif dans le déclenchement et le maintien de la chlorose. D'autre part, selon certains auteurs (Gouny \& Mazoyer, 1953; Evers, 1964; Clement et al., 1977; Le Tacon, 1976; Le Tacon et al., 1982; Jaillard, 1985), l'alimentation azotée, selon qu'elle est ammoniacale ou nitrique, pourrait favoriser le déclenchement de la chlorose.

Pour tenter de discriminer l'action du calcium de celle du couple bicarbonatecarbonate et apprécier les effets de la nutrition en azote et en fer, une série d'expériences de croissance de plants fut réalisée en faisant varier les facteurs suivants : l'apport en calcium, le rapport des ions $\mathrm{CO}_{3}^{2-} / \mathrm{HCO}_{3}^{-}$, la présence ou l'absence du $\mathrm{Fe}^{2+}$, et le type d'alimentation azotée.

Concrètement, des cultures de plants ont été faites en serre sur un support inerte (sable de Fontainebleau), de granulométrie moyenrie $200 \mu \mathrm{m}$ en présence de

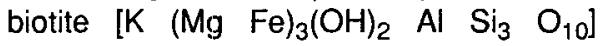
comme source de fer et d'une solution nutritive comme source d'éléments minéraux. Pour étudier l'influence du calcium et du carbonate-bicarbonate, le support a été enrichi ou non en carbonate de calcium (craie de granulométrie 20-50 $\mu \mathrm{m}$ ou en sulfate de calcium (gypse de granulométrie 20-50 $\mu \mathrm{m}$ ).

\section{MATÉRIEL ET MÉTHODES}

\section{Les traitements et le dispositif expéri- mental}

Nous avons effectué 12 traitements, chacun d'eux étant répété 10 fois (Tableau I). Des tubes en plastique de $5 \mathrm{~cm}$ de diamètre, $30 \mathrm{~cm}$ de long, fermés à la base par de la toile à bluter de $20 \mu \mathrm{m}$ de maille permettant la percolation des solutions d'arrosage, sont employés pour la culture.

Trois mélanges différents sont préparés : le premier est composé de $600 \mathrm{~g}$ de sable de Fontainebleau enrichi par $2 \mathrm{~g}$ de biotite. Le deuxième reçoit en plus $10 \%$ de carbonate de calcium. Le troisième contient $2 \mathrm{~g}$ de biotite et du gypse $\left(\mathrm{CaSO}_{4} 2 \mathrm{H}_{2} \mathrm{O}\right)$, dont la quantité est calculée d'une façon telle que chaque tube de culture renferme la même quantité de calcium que dans le mélange précédent. Ces substrats de culture sont disposés dans les tubes et supportent une plantule de deux ans provenant de pépinière. Les plants ont été cultivés à partir du 11 décembre 1985, arrosés quotidiennement par $10 \mathrm{ml}$ de solution nutritive au moyen d'un 
Tableau I. Description des 12 traitements effectués. Chaque traitement comporte 10 répétitions. $A=$ azote ammoniacal; $N=$ azote nitrique.

\begin{tabular}{lllll}
\hline $\mathrm{N}^{\circ}$ & $\mathrm{CaCO}_{3}$ & Gypse & $\mathrm{Fe}^{2+}$ & $\begin{array}{l}\text { Nutrition } \\
\text { azotée }\end{array}$ \\
\hline 1 & + & - & + & $\mathrm{A}$ \\
2 & + & - & - & $\mathrm{A}$ \\
3 & + & - & + & $\mathrm{N}$ \\
4 & + & - & - & $\mathrm{N}$ \\
5 & - & + & + & $\mathrm{A}$ \\
6 & - & + & - & $\mathrm{A}$ \\
7 & - & + & + & $\mathrm{N}$ \\
8 & - & + & - & $\mathrm{N}$ \\
9 & - & - & + & $\mathrm{A}$ \\
10 & - & - & - & $\mathrm{A}$ \\
11 & - & - & + & $\mathrm{N}$ \\
12 & - & - & + & $\mathrm{N}$ \\
\hline
\end{tabular}

système d'arrosage automatique. Ils se sont développés pendant une saison de végétation et ont été prélevés le 22 octobre 1986.

Deux solutions nutritives ont été préparées. Elles ne diffèrent que par la source d'azote, celui-ci étant fourni soit sous forme nitrique, soit sous forme ammoniacale (Tableau II). La solution ammoniacale contient en outre $4 \mathrm{mg} / \mathrm{lde}$ "N-Serv" (2-chloro-6-trichlorométhylpyridine) destiné à empêcher la nitrification. Ce composé organique n'a pas d'action sur le métabolisme des plantes et ne contient pas d'azote (Clement et al., 1977).

Les plants dont le traitement prévoit la présence de $\mathrm{Fe}^{2+}$ ont été arrosés une fois par semaine par $10 \mathrm{ml}$ de solution nutritive contenant $25 \mathrm{mg}$ de $\mathrm{FeSO}_{4} 7 \mathrm{H}_{2} \mathrm{O}$ par litre (environ 5 ppm de $\left.\mathrm{Fe}^{2+}\right)$. Les percolats sont récupérés dans des flacons de $250 \mathrm{ml}$ placés au-dessous des tubes de culture. Etant donné le très faible encombrement du dispositif ( 2 à $3 \mathrm{~m}^{2}$ au total) it n'a pas été jugé nécessaire de distribuer les traitements au hasard dans l'espace.

\section{Analyses des percolats}

Les analyses ont été faites sur des solutions filtrées, recueillies pendant un mois à la base des tubes. Afin de réduire l'activité biologique au minimum, les percolats furent stabilisés par 2 $\mathrm{ml}$ de chloroforme. Les analyses, portant sur 4 percolats (février, avril, juin, août 1986), concernaient les paramètres et éléments suivants :

\section{- Alcalinité et $p H$}

L'alcalinité, en meq/l a été calculée à partir des données du titrage d'une aliquote $(20 \mathrm{ml})$ jusqu'à $\mathrm{pH} 4,5$, par $\mathrm{HCl} 0,1 \mathrm{~N}$.

\section{- Azote ammoniacal}

L'analyse concernait les traitements ammoniacaux : le dosage de $\mathrm{NH}_{4}^{+}$fut effectué par colorimétrie du complexe bleu du sel de diazonium avec $\mathrm{NH}_{4}^{+}(\lambda=630 \mathrm{~nm})$.

\section{- Azote nitrique}

Les nitrates des percolats nitriques ont été réduits en nitrites par le sulfate d'hydrazine en milieu basique, en présence de sulfate de cuivre. Le dichlorure de naphtyléthylène diamine et la sulfanilamide forment, en présence de nitrites, un composé azoïque rose et stable avec le temps, identifié en colorimétrie $(\lambda=$ $520 \mathrm{~nm}$ ).

\section{- Autres éléments}

$K$ est dosé par spectrométrie d'absorption atomique alors que $\mathrm{P}, \mathrm{S}, \mathrm{Ca}, \mathrm{Mg}$ et $\mathrm{Fe}$ le sont par

Tableau II. Composition élémentaire des solutions nutritives exprimée en $\mathrm{mg} / \mathrm{l}$ (d'après les recommandations de Gouny et Mazoyer, 1953).

\begin{tabular}{llllllllllllll}
\hline & $N$ & $P$ & $K$ & $C a$ & $M g$ & $S$ & $M n$ & $Z n$ & $B$ & $C u$ & $M o$ & $C l$ & $N a$ \\
\hline $\mathrm{NH}_{4}^{+}$ & 33,6 & 44,64 & 56,16 & 48 & 9,6 & 38,4 & 0,19 & 0,17 & 0,13 & 0,04 & 0,097 & 113,6 & 0 \\
$\mathrm{NO}_{3}^{4}$ & 33,6 & 44,64 & 56,16 & 48 & 9,6 & 38,4 & 0,19 & 0,17 & 0,13 & 0,04 & 0,097 & 25,4 & 55,2 \\
\hline
\end{tabular}


spectrométrie d'émission atomique (torche à plasma).

Les calculs statistiques portant sur les comparaisons de moyennes sont effectués selon le test de Student modifié, dit test de Unfal et de Bonferroni (Bachacou et al., 1981).

\section{Analyses des plantes}

En fin d'expérience, l'état des plants fut observé et leurs caractères morphologiques soigneusement établis et notés. Ainsi 7 classes de plants ont été distinguées, en fonction de l'intensité de la chlorose et de la taille des plants :

1) les plants sains à croissance normale,

2) les plants sains à croissance réduite : la longueur des nouvelles pousses est inférieure à celle de la classe précédente, aucun symptôme chlorotique n'étant apparu sur les plants;

3) les plants légèrement chlorosés à croissance normale,

4) les plants légèrement chlorosés à croissance réduite,

5) les plants chlorosés à croissance normale,

6) les plants chlorosés à croissance réduite,

7) les plants morts.

La croissance est qualifiée de "normale" ou de "réduite" par comparaison du nombre et de la longueur des nouvelles pousses de chaque plant avec ceux des plants des témoins ammoniacaux.

Remarque : certains plants ont péri accidentellement sous l'effet d'un choc thermique entraîné par un fort rayonnement solaire survenu en serre, les 1 or et 2 mai 1986. C'est la raison pour laquelle nous négligerons le nombre des plants morts lors de l'interprétation de nos résultats.

Pour ce qui concerne les analyses, les plants ont été assemblés en 6 groupes. Tous les plants morts ont été écartés. Chaque groupe est constitué par l'ensemble des plants des traitements qui ne different que par l'apport de solution ferreuse. Ainsi, par exemple, les plants non morts, soumis au traitement nutritionnel ammoniacal, sur support carbonaté, forment un groupe unique, que les traitements aient reçu ou non du fer en solution. En fin d'expérience, les aiguilles jeunes des plants de chaque groupe sont coupées, mélangées, séchées à $60^{\circ} \mathrm{C}$ pendant $48 \mathrm{~h}$ et pesées. II en va de même des racines, préalablement lavées et dont on n'a collecté que celles dont le diamètre est inférieur à $2 \mathrm{~mm}$.

Les analyses des éléments totaux ont été faites sur les poudres des aiguilles broyées et des racines dont on a déterminé le taux de matière sèche. L'analyse de $\mathrm{P}, \mathrm{S}, \mathrm{Ca}, \mathrm{Mg}, \mathrm{K}, \mathrm{Fe}$ et $\mathrm{Mn}$ fut faite après minéralisation perchlorique pratiquée selon le protocole de Clement (1977). Tous ces éléments ont été dosés à la torche à plasma, sauf $K$ dosé en absorption atomique. L'analyse de l'azote fut effectuée après minéralisation sulfurique et dosage colorimétrique de l'ion $\mathrm{NH} 4+$ produit (Clement, 1977). La solubilité des éléments fult déterminée à partir d'aiguilles ou de racines séchées, broyées dans l'eau pendant 3 à $4 \mathrm{~min}$ (rapport matériel/eau de 2 à $5 \mathrm{~g}$ pour $50 \mathrm{ml}$ ). Après centrifugation et filtration, les solutions ont subi une minéralisation, soit perchlorique pour le dosage de $\mathrm{P}, \mathrm{S}, \mathrm{Ca}, \mathrm{Mg}, \mathrm{K}, \mathrm{Fe}$ et $\mathrm{Mn}$, soit sulfurique pour le dosage de l'azote. Le fer extractible à l'acide chlorhydrique $\mathrm{iN} \mathrm{a}$ été déterminé selon la méthode de Oserkowsky (1933) : $1 \mathrm{~g}$ de poudre d'aiguilles ou de racines est agité $24 \mathrm{~h}$ avec $25 \mathrm{ml}$ HCL N par retournement. Le fer est dosé à la torche à plasma après centrifugation et filtration de la suspension.

Remarque : l'insuffisance de matériel foliaire ou racinaire récolté pour chaque traitement n'a pas permis d'effectuer des répétitions d'analyses qui sont donc données sans marge statistique.

\section{Isotopes stables $\left({ }^{13} \mathrm{C} /{ }^{2} \mathrm{C}\right) \mathrm{du}$ carbone des carbonates}

Pour savoir si le $\mathrm{CaCO}_{3}$ associé aux racines résulte d'une précipitation à partir de $\mathrm{Ca}\left(\mathrm{HCO}_{3}\right)_{2}$ ou bien d'un lavage inefficace laissant de la craie collée aux racines, la mesure des isotopes stables $\left(\delta^{13} C\right)$ est précieuse. Malheureusement, l'insuffisance de matériel racinaire nous a conduit à regrouper les racines des plants poussant en présence de $\mathrm{CaCO}_{3}$ et $\mathrm{CaSO}_{4}$. Le $\mathrm{CO}_{2}$ du $\mathrm{CaCO}_{3}$ a été déplacé sous vide par $\mathrm{HCl}$ et piégé dans une solution de soude. Aprés ajout de $\mathrm{SrCl}_{2}$ on produit du $\mathrm{SrCO}_{3}$ dont les isotopes du carbone furent analysés en spectrométrie de masse. La craie utilisée dans les expériences a subi le même traitement. 


\section{RÉSULTATS ET DISCUSSION}

\section{Analyse des percolats}

Nous présenterons uniquement les résultats d'analyses du deuxième percolat (avril 1986), et cela, uniquement pour des plants ne recevant pas de $\mathrm{FeSO}_{4}$, car nous n'avons pas trouvé de différence entre traitements recevant du $\mathrm{Fe}^{2+}$ et ceux qui en sont exempts. Ce fut aussi la raison pour laquelle les plants ont été regroupés pour l'analyse foliaire en lots comprenant les traitements avec ou sans $\mathrm{FeSO}_{4}$. Par

Tableau III. Analyse du deuxième percolat (avril 1986) : valeurs moyennes et écarts types du pH, de l'alcalinité et des concentrations.

\begin{tabular}{|c|c|c|c|c|c|c|c|c|c|c|c|}
\hline & & $N^{\circ}$ & $p H$ & $\begin{array}{l}\text { Alcalinité } \\
\text { me.t-1 }\end{array}$ & $N$ & $P$ & $\begin{array}{l}K \\
m g . H^{-1}\end{array}$ & $S$ & $\mathrm{Ca}$ & $M g$ & $\begin{array}{l}F e \\
\mu g . /-1\end{array}$ \\
\hline & $\mathrm{CaCO}_{3}$ & 2 & $\begin{array}{l}7,91 \\
0,192\end{array}$ & $\begin{array}{l}1,72 \\
0,494\end{array}$ & $\begin{array}{c}22,7 \\
3,57\end{array}$ & $\begin{array}{l}5,6 \\
1,79\end{array}$ & $\begin{array}{r}66,9 \\
3,8\end{array}$ & $\begin{array}{r}53,1 \\
4,31\end{array}$ & $\begin{array}{l}98,5 \\
14,54\end{array}$ & $\begin{array}{c}15,0 \\
0,85\end{array}$ & $\begin{array}{l}93 \\
24,1\end{array}$ \\
\hline \multirow[t]{3}{*}{$\mathrm{NH}_{4}^{+}$} & $\mathrm{CaSO}_{4}$ & 6 & $\begin{array}{l}7,36 \\
0,352\end{array}$ & $\begin{array}{l}0,43 \\
0,171\end{array}$ & $\begin{array}{c}30,1 \\
7,77\end{array}$ & $\begin{array}{c}18,0 \\
1,13\end{array}$ & $\begin{array}{c}59,6 \\
5,89\end{array}$ & $\begin{array}{c}548,9 \\
14,93\end{array}$ & $\begin{array}{c}614,8 \\
11,10\end{array}$ & $\begin{array}{c}17,3 \\
1,29\end{array}$ & $\begin{array}{c}117 \\
49,5\end{array}$ \\
\hline & Témoin & 10 & $\begin{array}{l}5,33 \\
1,323\end{array}$ & $\begin{array}{l}0,25 \\
0,207\end{array}$ & $\begin{array}{l}26,3 \\
13,08\end{array}$ & $\begin{array}{c}44,3 \\
8,13\end{array}$ & $\begin{array}{c}61,8 \\
7,45\end{array}$ & $\begin{array}{c}53,1 \\
4,27\end{array}$ & $\begin{array}{c}92,0 \\
5,61\end{array}$ & $\begin{array}{c}18,0 \\
1,33\end{array}$ & $\begin{array}{l}72 \\
23,8\end{array}$ \\
\hline & $\mathrm{CaCO}_{3}$ & 4 & $\begin{array}{l}8,23 \\
0,284\end{array}$ & $\begin{array}{l}3,36 \\
1,044\end{array}$ & $\begin{array}{l}33,7 \\
5,55\end{array}$ & $\begin{array}{c}11,3 \\
1,84\end{array}$ & $\begin{array}{c}66,5 \\
4,22\end{array}$ & $\begin{array}{c}65,6 \\
4,83\end{array}$ & $\begin{array}{l}56,9 \\
18,47\end{array}$ & $\begin{array}{c}12,5 \\
1,03\end{array}$ & $\begin{array}{l}39 \\
17,0\end{array}$ \\
\hline \multirow[t]{8}{*}{$\mathrm{NO}_{3}^{-}$} & CaSO4 & 8 & $\begin{array}{l}7,83 \\
0,609\end{array}$ & $\begin{array}{l}0,96 \\
0,143\end{array}$ & $\begin{array}{l}27,6 \\
4,98\end{array}$ & $\begin{array}{c}15,8 \\
0,41\end{array}$ & $\begin{array}{c}53,9 \\
4,25\end{array}$ & $\begin{array}{r}558,2 \\
6,60\end{array}$ & $\begin{array}{c}573,6 \\
10,43\end{array}$ & $\begin{array}{c}14,9 \\
1,03\end{array}$ & $\begin{array}{l}79 \\
21,3\end{array}$ \\
\hline & Témoin & 12 & $\begin{array}{l}7,66 \\
0,159\end{array}$ & $\begin{array}{l}1,77 \\
0,225\end{array}$ & $\begin{array}{c}29,5 \\
3,24\end{array}$ & $\begin{array}{c}40,5 \\
5,96\end{array}$ & $\begin{array}{l}55,6 \\
2,119\end{array}$ & $\begin{array}{l}64,4 \\
1,51\end{array}$ & $\begin{array}{l}38,8 \\
10,77\end{array}$ & $\begin{array}{c}12,5 \\
0,49\end{array}$ & $\begin{array}{l}42 \\
17,5\end{array}$ \\
\hline & & 2 et 6 & & $\star *$ & & $* *$ & & ** & ** & $* \star$ & $* \star$ \\
\hline & & 2 et 10 & "* & $\star \star$ & & ** & & & & ** & \\
\hline & & 6 et 10 & "* & & & $* *$ & & 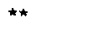 & "* & $\star \star$ & \\
\hline & & 4 et 8 & & $* \star$ & & & ** & ** & ** & $\star \star$ & \\
\hline & & 4 et 12 & & $* \star$ & & $\star \star$ & $* \star$ & & & & \\
\hline & & 8 et 12 & & $\star \star *$ & & $* *$ & & $\star \star *$ & $\star \star$ & $\star \star$ & \\
\hline \multirow{2}{*}{\multicolumn{2}{|c|}{$\begin{array}{l}\text { Compar. } \\
\text { des } \\
\text { moyennes } \\
\text { ** Dif. à } 1 \%\end{array}$}} & 2 et 4 & & ** & * & & & * & & ** & \\
\hline & & 2 et 8 & & * & & $* \star$ & $* \star$ & ** & ** & & \\
\hline \multirow[t]{7}{*}{ “Dif. à } & $5 \%$ & 2 et 12 & & & & $\star \star *$ & $\star \star \star$ & & $\star *$ & $\star \star$ & \\
\hline & & 6 et 4 & & ** & & * & & $* *$ & "* & $\star *$ & $* *$ \\
\hline & & 6 et 8 & & & & & & & & ** & \\
\hline & & 6 et 12 & & $\star \star \star$ & & ** & & ** & ** & ** & $\star \star$ \\
\hline & & 10 et 4 & ** & $* *$ & & $* *$ & & $*$ & & $* \star$ & \\
\hline & & 10 et 8 & ** & & & ** & * & $* *$ & "** & $* *$ & \\
\hline & & 10 et 12 & $\star *$ & ** & & & & & $\star$ & $\star \star *$ & \\
\hline
\end{tabular}


ailleurs, les analyses des percolats recueillis en février, juin et août s'avèrent très voisines (Khalil, 1987).

\section{pH et alcalinité}

Le Tableau III montre une différence significative de $\mathrm{pH}$ entre le témoin ammoniacal et tous les traitements nitriques. Le $\mathrm{pH}$ tend aussi à être plus élevé dans les percolats nitriques que dans les percolats ammoniacaux des autres traitements. La même remarque peut être faite si on compare les valeurs de l'alcalinité. En effet, l'alcalinité reflète en partie les échanges d'ions qui se produisent au niveau de la racine lors de l'absorption. Cette différence d'alcalinité est sans doute explicable par l'absorption active des ions azotés (Callot et al., 1982; Jaillard, 1985). En effet, quand la plante absorbe un cation ammonium $\left(\mathrm{NH}_{4}^{+}\right)$, elle libère dans le milieu un proton $\left(\mathrm{H}^{+}\right)$, ce qui abaisse le $\mathrm{pH}$. En revanche, quand elle absorbe un anion nitrate, elle libère un anion hydroxyl $\left(\mathrm{OH}^{-}\right)$ou bicarbonate $\left(\mathrm{HCO}_{3}^{-}\right)$: le $\mathrm{pH}$ s'élève.

Il est clair, en tout cas, que comparées au traitement sulfate, les plus fortes valeurs de l'alcalinité des traitements carbonatés, témoignent, à $\mathrm{pH}$ sensiblement voisins, d'une plus forte abondance d'ions $\mathrm{CO}_{3}^{2} / \mathrm{HCO}_{3}^{-}$dans les solutions.

\section{Eléments}

\section{L'azote}

La seule différence significative concerne les teneurs en azote des percolats des traitements (2) et (4) (Tableau I) comportant du carbonate de calcium. Lorsque l'alimentation des plants se fait sous forme nitrique, les percolats comportent plus d'azote. On pourrait en déduire qu'en présence de carbonate de calcium, les plants prélèvent davantage d'azote sous forme ammoniacale que sous forme nitrique.

\section{Le phosphore}

Quel que soit le mode de nutrition azotée, la concentration en phosphore des percolats s'abaisse considérablement quand les supports de culture contiennent du calcium sous forme de sulfate ou de carbonate. On ne peut expliquer les faibles teneurs que par un processus de rétention physico-chimique du phosphore sur les composés minéraux calciques.

\section{Le potassium}

Les concentrations en potassium des percolats étant significativement plus élevées dans les traitements comportant du $\mathrm{CO}_{3} \mathrm{Ca}$, on peut en déduire que ce support carbonaté laisse mieux filtrer le potassium ou bien que l'élément est moins absorbé par les plants. Nous verrons, par l'analyse des plants, que cette seconde hypothèse se trouve être vérifiée.

\section{Le soufre}

II faut d'abord considérer les traitements effectués en présence de gypse. Les hautes teneurs en soufre et en calcium sont indiscutablement dues à la solubilisation du sulfate ce calcium. Ces traitements mis à part, nous observons que les percolats de l'alimentation nitrique renferment plus de soufre que les percolats homologues du système d'alimentation ammoniacal. On en déduit que les plants alimentés en azote nitrique ont absorbé moins de 
Tableau IV. Description des plantes des différents traitements et dénombrement.

\begin{tabular}{|c|c|c|c|c|c|c|c|c|c|}
\hline & & & $\begin{array}{l}\text { Saines } \\
\text { Croissance } \\
\text { normale }\end{array}$ & $\begin{array}{l}\text { Saines } \\
\text { Croissance } \\
\text { réduite }\end{array}$ & $\begin{array}{l}\text { Légère } \\
\text { chlorose } \\
\text { Croissance } \\
\text { normale }\end{array}$ & $\begin{array}{l}\text { Légère } \\
\text { chlorose } \\
\text { Croissance } \\
\text { réduite }\end{array}$ & $\begin{array}{l}\text { Chlorosées } \\
\text { Croissance } \\
\text { normale }\end{array}$ & $\begin{array}{l}\text { Chlorosées } \\
\text { Croissance } \\
\text { réduite }\end{array}$ & Mortes \\
\hline & $\mathrm{CaCO}_{3}$ & $+\mathrm{Fe}$ & 2 & 0 & 1 & 1 & 3 & 1 & 2 \\
\hline & & - & 2 & 0 & 0 & 2 & 3 & 1 & 2 \\
\hline \multirow[t]{6}{*}{$\mathrm{NH}_{4}^{+}$} & $\mathrm{CaSO}_{4}$ & $+\mathrm{Fe}$ & 6 & 3 & 1 & 0 & 0 & 0 & 0 \\
\hline & & - & 3 & 6 & 0 & 0 & 0 & 0 & 1 \\
\hline & Témoin & $+\mathrm{Fe}$ & 10 & 0 & 0 & 0 & 0 & 0 & 0 \\
\hline & & - & 10 & 0 & 0 & 0 & 0 & 0 & 0 \\
\hline & $\mathrm{CaCO}_{3}$ & $+\mathrm{Fe}$ & 0 & 0 & 0 & 0 & 10 & 0 & 0 \\
\hline & & - & 0 & 0 & 0 & 0 & 7 & 3 & 0 \\
\hline \multirow[t]{4}{*}{$\mathrm{NO}_{3}^{-}$} & $\mathrm{CaSO}_{4}$ & $+\mathrm{Fe}$ & 0 & 0 & 0 & 0 & 3 & 4 & 3 \\
\hline & & - & 0 & 0 & 0 & 0 & 0 & 6 & 4 \\
\hline & Témoin & $+\mathrm{Fe}$ & 0 & 0 & 0 & 0 & 10 & 0 & 0 \\
\hline & & - & 0 & 0 & 0 & 0 & 10 & 0 & 0 \\
\hline
\end{tabular}

soufre que les plants nourris d'azote ammoniacal. On peut penser que la plus faible absorption de soufre en milieu nitrique est occasionnée par une compétition d'anions $\mathrm{SO}_{4}^{2-} / \mathrm{NO}_{3}^{-}$.

\section{Le calcium}

Les percolats ammoniacaux contiennent plus de calcium que les percolats nitriques. Cela s'explique de deux façons: d'abord la solubilité du carbonate de calcium est certainement plus faible dans les traitements nitriques, qui ont des $\mathrm{pH}$ plus élevés; en outre, en l'absence de carbonate de calcium, l'absorption de calcium par les plants est plus importante en nutrition nitrique qu'en nutrition ammoniacale. Nous le monterons ultérieurement à propos de l'analyse des racines.
Le fer et le magnésium

Les différences de concentration en fer et en magnésium selon les traitements nous semblent être liées à la solubilisation de la biotite ou à des impuretés (notamment en ce qui concerne le fer) présentes dans le gypse ou la calcite.

\section{Description des plants}

En fin d'expérience, et pour chaque traitement, la distribution des plants en classes différentes s'établit de la façon indiquée dans le Tableau IV. La comparaison des plants poussant sur support carbonaté ou gypseux nous permet d'insister sur le fait que les anions $\mathrm{CO}_{3}^{2-} / \mathrm{HCO}_{3}^{-}$sont le uprinci- 
pe actif» du carbonate de calcium dans la chlorose. En effet, ce n'est pas le cation calcium qui est en lui-même responsable de la chlorose car, si cela était, les symptômes chlorotiques seraient plus accusés dans les traitements avec gypse, qui est plus soluble que le calcaire. Il peut provoquer des diminutions de croissance, mais, par sa seule présence, il ne peut pas déclencher les symptômes chlorotiques.

D'autre part, il semble que la nutrition ammoniacale convient mieux au sapin de Nordmann que la nutrition nitrique qui provoque de graves symptômes chlorotiques. Ce résultat corrobore les données de Le Tacon (1976), Clement et al., (1977); Le Tacon et al., (1982) sur l'épicéa et le pin noir. Mais il s'oppose à celle de Gouny \& Mazoyer (1953) sur le lupin jaune.

\section{Analyse des plants}

\section{La croissance des plants}

Si l'on se réfère au poids des nouvelles pousses et des racines, il apparaît que la

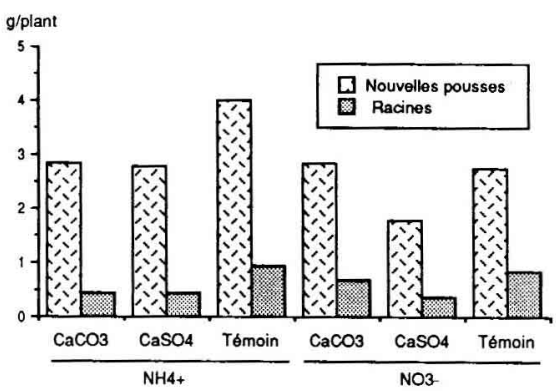

Fig. 1. Croissance des plants : poids des nouvelles pousses fraîches et des racines sèches de diamètre inférieur à $2 \mathrm{~mm}$ ( $g /$ plant).
A

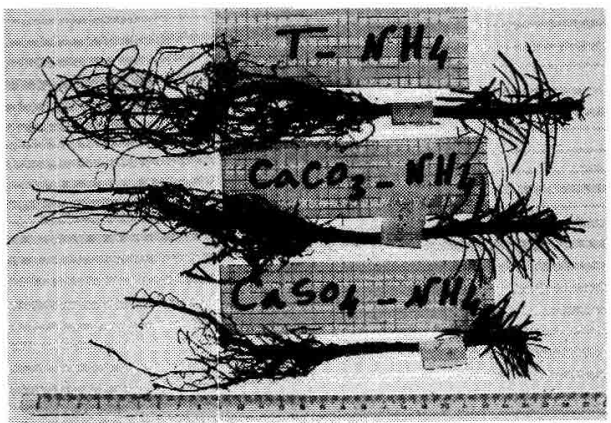

B

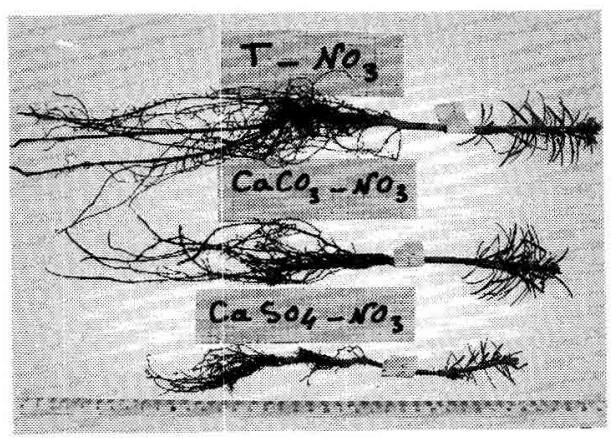

Fig. 2A et B. Plants cultivés en présence de solutions nutritives. Comparaison de la croissance des racines en système de nutrition ammoniacale (Fig. 2A) et nitrique (Fig. 2B).

présence de calcium dans les substrats affecte le développement de la biomasse racinaire (Figs. 1 et 2) et aussi généralement la biomasse foliaire (Fig. 1). On observera également (Fig. 1) que le développement de la biomasse foliaire est meilleur dans le traitement ammoniacal dont le support est exempt de calcium, que dans son homologue nitrique. La comparaison des poids des nouvelles pousses des traitements témoins est en effet à l'avantage de l'alimentation ammoniacale (4,013 g/plante) contre 2,755 pour l'alimentation nitrique. 


\section{Les éléments (tableaux V et VI)}

L'azote

II nous semble important de souligner que les teneurs en azote des plants des traitements nitriques sont indifférentes à la présence ou à l'absence de carbonate de calcium. Ce qui prime, c'est avant tout une médiocre alimentation en azote lorsque la seule source d'azote est nitrique. Le déficit azoté des plants des traitements nitriques fut observé par Le Tacon (1976) et Le Tacon et al. (1982) sur le pin noir et l'épicéa. Ce résultat met en évidence le caractère «nitratophobe» du sapin de Nordmann.

\section{Le phosphore}

Il faut insister sur le fait que, dans l'absolu, les teneurs en phosphore, même en présence de carbonate de calcium, sont élevées. Aussi, la moindre absorption de phosphore dans les plants chlorosés n'est pas cause de chlorose. La forte teneur en phosphore des aiguilles vertes des traitements ammoniacaux peut, en revanche, paraître anormale mais pourrait s'expliquer par le fait que la nutrition azotée est uniquement cationique $\left(\mathrm{NH}_{4}^{+}\right)$, ce qui renforcerait les prélèvements d'anions dont les $\mathrm{PO}_{4}^{3-}$.

\section{Le soufre}

Les moindres teneurs en soufre des aiguilles chlorosées confirment les résultats obtenus en forêt de Greffeil (Khalil, 1987). Mais, là aussi, la teneur en soufre total reste normale et c'est peut-être plus le rapport $S / N$ et la teneur un peu plus faible en soufre soluble qui se corrèlent à la chlorose.
Le calcium

La présence de minéraux calciques (carbonate ou sulfate) dans les substrats a
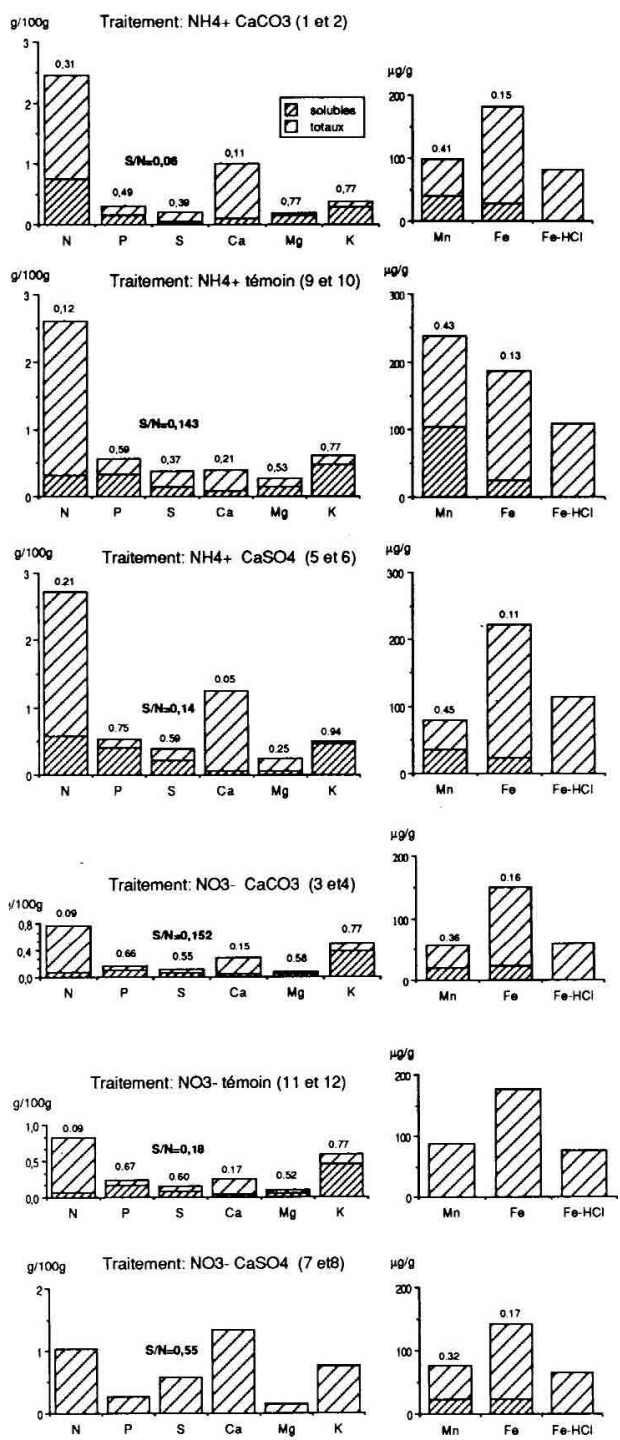

Fig. 3. Eléments totaux et éléments solubles des aiguilles jeunes (barres) et rapports entre éléments solubles et éléments totaux (chiffres figurant au-dessus des barres). 
élevé la rétention du calcium dans les plants (Figs. 3 et 4 ).

En ce qui concerne les racines (Fig. 4), tous les traitements, excepté le témoin ammoniacal (9-10) mais y compris le témoin nitrique $(11-12)$, conduisent à la
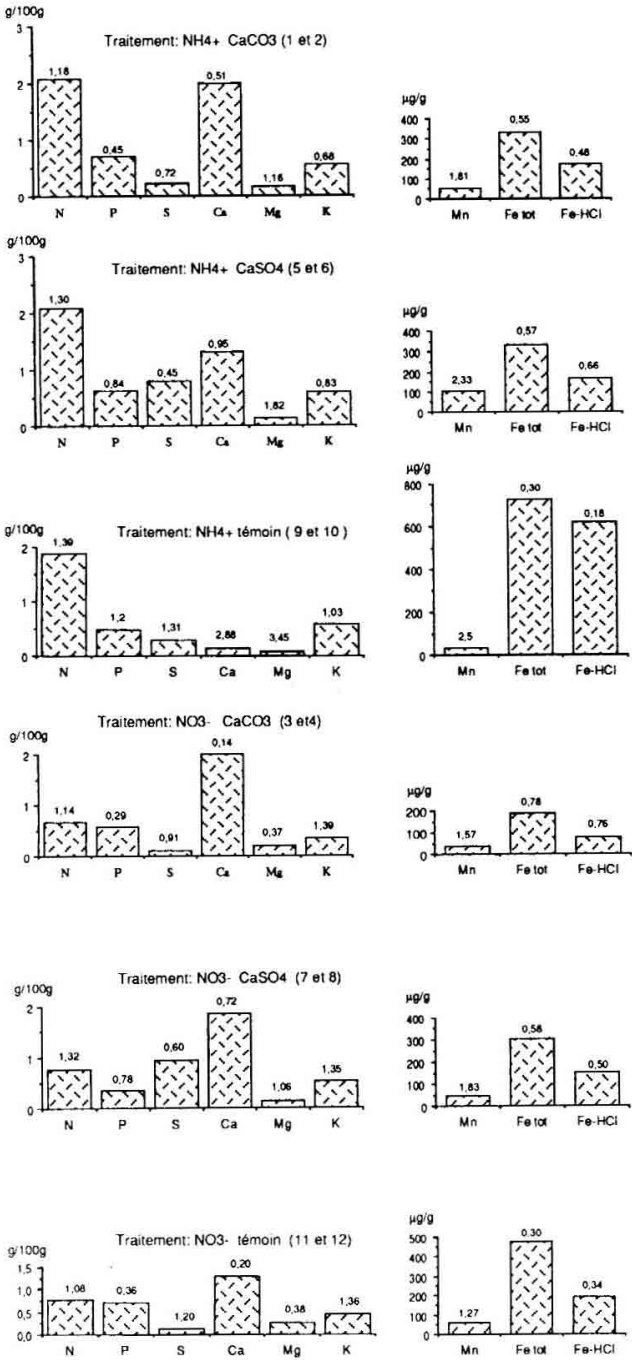

Fig. 4. Composition élémentaire des racines (barres) et rapports entre éléments totaux des aiguilles et éléments totaux des racines (chiffres portés au-dessus des barres)

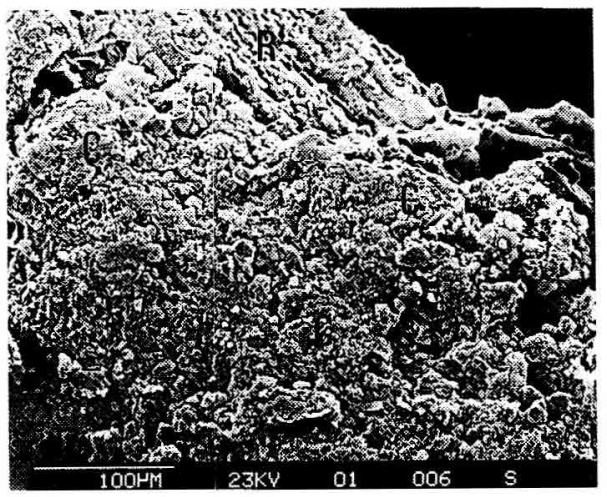

Fig. 5. Observations au microscope électronique à balayage des racines des plants témoins alimentés en azote nitrique : vue des enduits carbonatés $(C)$ recouvrant partiellement les tissus végétaux racinaires (R).

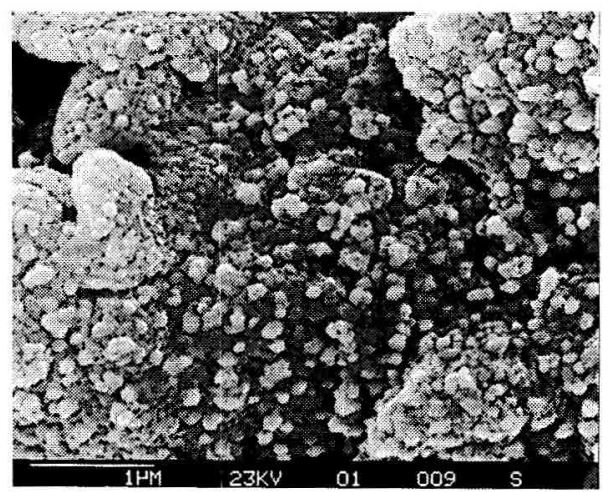

Fig. 6. MEB : Détail des enduits carbonatés révélant des aspérités qui seraient les germes de croissance de minéraux carbonatés (sans doute calcite).

rétention du calcium, par ailleurs peu soluble. Des coupes, effectuées sur les racines ne montrent pas de précipitation intracellulaire de sels de calcium comme cela fut observé par Jaillard (1985) chez certaines graminées. En revanche, les racines s'entourent par endroits d'un manchon de carbonate de calcium (Fig. 5) sans forme cristalline nette mais montrant des aspérités (Fig. 6) qui apparaissent 
comme des germes de croissance de cristaux de calcite.

L'analyse isotopique du carbone des carbonates prouve que ces enduits racinaires résultent de la précipitation du carbonate et non de l'attachement de particules de craie. La craie, en effet, et le $\mathrm{SrCO}_{3}$ qui en est issu, ont un $\delta^{13} \mathrm{C}$ respectivement de $1,0 \%$ et $-1,2 \%$ o tandis que les enduits racinaires ont un $\delta^{13} \mathrm{C}$ de $-11,7 \%$. Cette valeur souligne la contribution d'un carbone d'origine biologique qui n'est autre que le $\mathrm{CO}_{2}$ rhizosphérique produit par la respiration des racines et la dégradation des exsudats et dont le $\delta^{13} \mathrm{C}$ est de l'ordre de $-25 \%$ (Deines, 1980).

L'équation réversible de dissolution et reprécipitation de $\mathrm{CaCO}_{3}$ donne la clé de l'interprétation :

$\mathrm{CO}_{2}+\mathrm{H}_{2} \mathrm{O}+\mathrm{CaCO}_{3} \underset{(2)}{\stackrel{(1)}{=}} \mathrm{Ca}^{2+}+2 \mathrm{HCO}_{3}^{-}$

Dans le sens (1), à l'équilibre, du $\mathrm{CO}_{2}$ au $\delta^{13} \mathrm{C}$ de $-25 \%$ agit sur la craie à $1,0 \%$ 。 pour donner du bicarbonate à $-13 \%$ o. La précipitation du $\mathrm{CaCO}_{3}$ à partir du bicarbonate (sens (2)) crée un fractionnement de l'ordre de $+1,8 \%$ (Emrich et al., 1970), de sorte que le $\mathrm{CaCO}_{3}$ précipite, à l'équilibre, avec un $\delta^{13} \mathrm{C}$ de l'ordre de $-11 \%$.

Pour ce qui concerne les aiguilles (Fig. 3) l'absorption de calcium a été stimulée par les substrats calciques, sauf dans le traitement nitrique avec $\mathrm{CaCO}_{3}(3$ et 4). Les basses teneurs en calcium des aiguilles dans ce traitement et aussi dans le témoin ( 11 et 12) paraissent liées à la faible mobilité de l'élément comme l'illustrent bien les valeurs minima du rapport des teneurs entre aiguilles et racines (Fig. 4).

II est patent que lorsque le milieu de culture renferme des minéraux calciques, le sapin de Nordmann bloque le calcium au niveau de ses racines et ne parvient pas à réguler l'absorption de cet élément.
Le magnésium

L'absorption du magnésium, ainsi que son transfert entre racines et parties aériennes sont fortement atténués en présence de carbonate de calcium, mais les teneurs restent, dans l'absolu, tout à fait suffisantes, sauf pour les traitements ( 3 et 4 ). On remarque également une absorption et une mobilité moindre de cet élément, en condition de nutrition nitrique.

\section{Le potassium}

Dans tous les cas, la présence de carbonate de calcium paraît déprimer l'absorption foliaire du potassium, notamment lors des traitements ammoniacaux. En effet, les aiguilles chlorosées des traitements 1 et 2 contiennent moins de potassium que les aiguilles vertes des traitements recevant du gypse (5 et 6) ou témoins (9 et 10). Ces faits s'opposent à ceux observés par Le Tacon (1976) sur l'épicéa et le pin noir, par Prison et al., (1982) sur le peuplier et Anderson (1982) sur l'eucalyptus, mais corroborent les résultats obtenus sur les plants du sapin de Nordmann de la forêt de Greffeil (Khalil, 1987; Khalil et al., 1988).

D'autre part, le transfert du potassium entre les racines et les parties aériennes est très affecté par la présence de $\mathrm{CO}_{3} \mathrm{Ca}$ dans le cas d'une alimentation ammoniacale. On remarquera en effet la chute du rapport des teneurs en $K$ des aiguilles et racines entre le traitement témoin et le traitement avec support carbonaté (Fig. 4).

\section{Le manganèse}

Les teneurs en manganèse des aiguilles ne représentent pas de grandes différences entre les divers traitements, à 
l'exception des aiguilles des traitements 5 et 6 qui montrent des teneurs très élevées. La mobilité du manganèse dans les plants des traitements ammoniacaux est négativement affectée par la présence de carbonate de calcium dans les substrats.

\section{Le fer}

Les teneurs en fer total sont plus élevées dans les plants verts que dans les plants chlorosés. Cette caractéristique est encore plus évidente quand on compare les teneurs en fer extractible à l'acide chlorhydrique $\mathrm{N}$ des aiguilles qui sont plus élevées dans les traitements ammoniacaux que dans les traitements nitriques. Par ailleurs, l'effet du carbonate est très sensible en alimentation ammoniacale mais minime en alimentation nitrique. On notera également que le transfert du fer vers les parties aériennes semble plus efficace quand les substrats renferment du carbonate de calcium ou du sulfate de calcium. Plus qu'une question de transport, c'est donc une question d'absorption et peutêtre aussi de réduction si l'on admet que $\mathrm{HCl}$ extrait de préférence le fer ferreux (Zech, 1970).

\section{CONCLUSION}

De nombreux conifères sont bien connus pour être calcifuges : l'épicéa commun étudié par Le Tacon (1976) et Clement (1977); le pin sylvestre, pour lequel les travaux de Zech (1970) ont mis en évidence l'intérêt du dosage du fer actif dans la caractérisation de la chlorose calcaire; le pin maritime, et plus encore, le Douglas. Toutes ces essences présentent des symptômes de chlorose plus ou moins graves en milieu calcaire. Le cas des sapins a été, semble-t-il, moins étudié. Fiedler (1973), citant les travaux de Rehfuess (1967) et de Zech (1970) estime que le sapin pectiné (Abies alba Mill.) est légèrement calcifuge. II n'y a pas de travaux, à notre connaissance, concernant les autres espèces du genres Abies. C'est pourquoi le cas du sapin de Nordmann était intéressant à étudier.

Dans les expériences en solutions nutritives qui ont été relatées, il apparaît nettement que la chlorose du sapin de Nordmann peut survenir de deux manières :

\section{Par excès de carbonate de calcium en situation d'alimentation azotée ammo- niacale}

En effet, la croissance des parties aériennes, comme celle des racines, est dans ce cas fortement réduite. II apparaît aussi que les anions bicarbonate-carbonate sont alors directement en cause puisque la croissance et la vigueur ne sont pas déprimées par la présence de sulfate de calcium.

Les plants chlorosés se caractérisent par des diminutions importantes de la quantité de soufre soluble dans les aiguilles et du rapport $S / N$, liées d'ailleurs à une faible teneur en azote soluble. Ceci laisse supposer que des difficultés dans le métabolisme du soufre constituent un facteur limitant de la croissance.

L'absorption du fer et du manganèse ainsi que le transfert de ce dernier des racines aux aiguilles sont limités mais ne conduisent cependant pas à des teneurs excessivement faibles. Même la teneur du fer «actif» (extrait par $\mathrm{HCl}$ ), tout en étant effectivement plus faible en présence de $\mathrm{CaCO}_{3}$, n'atteint jamais de très faibles valeurs. 


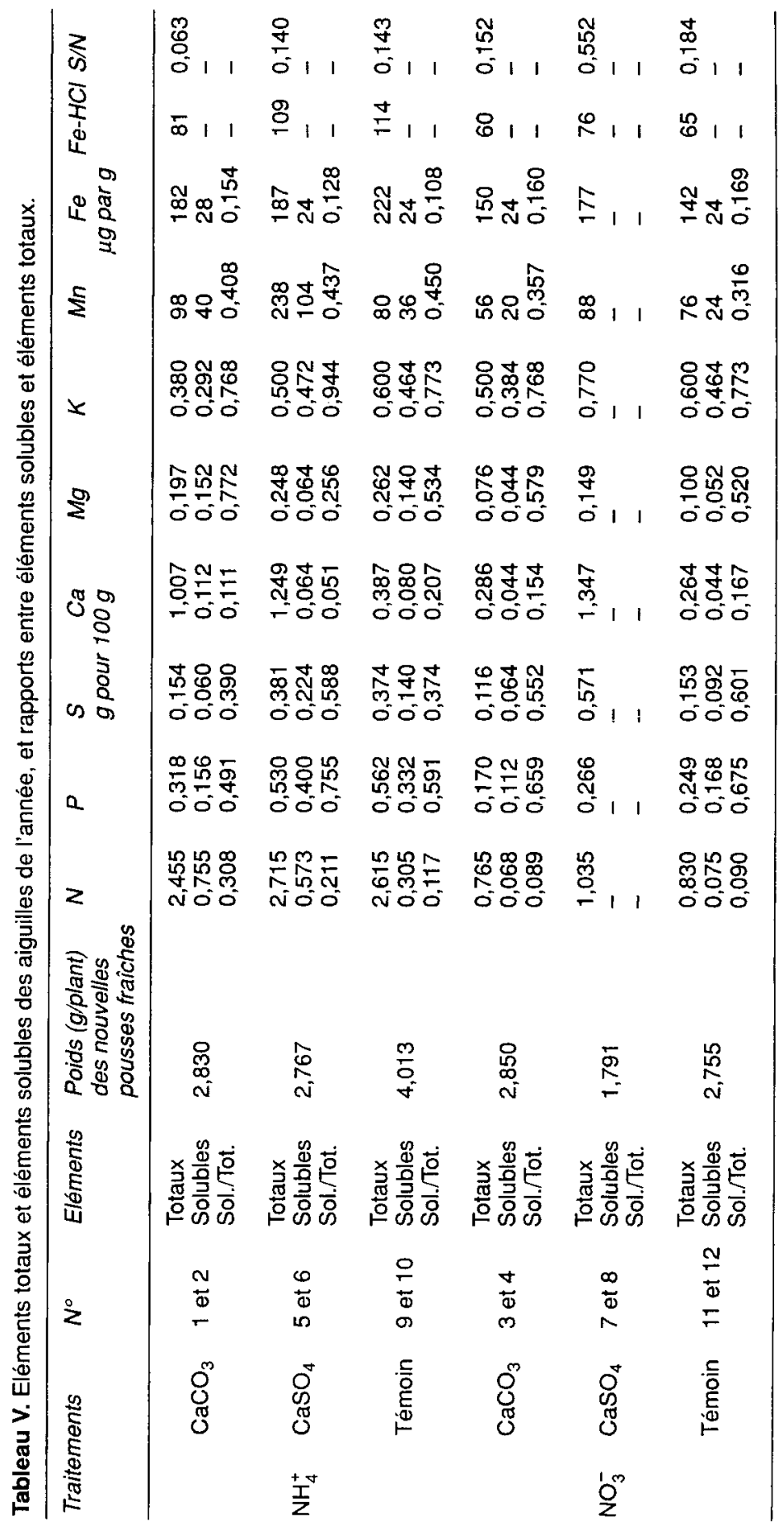




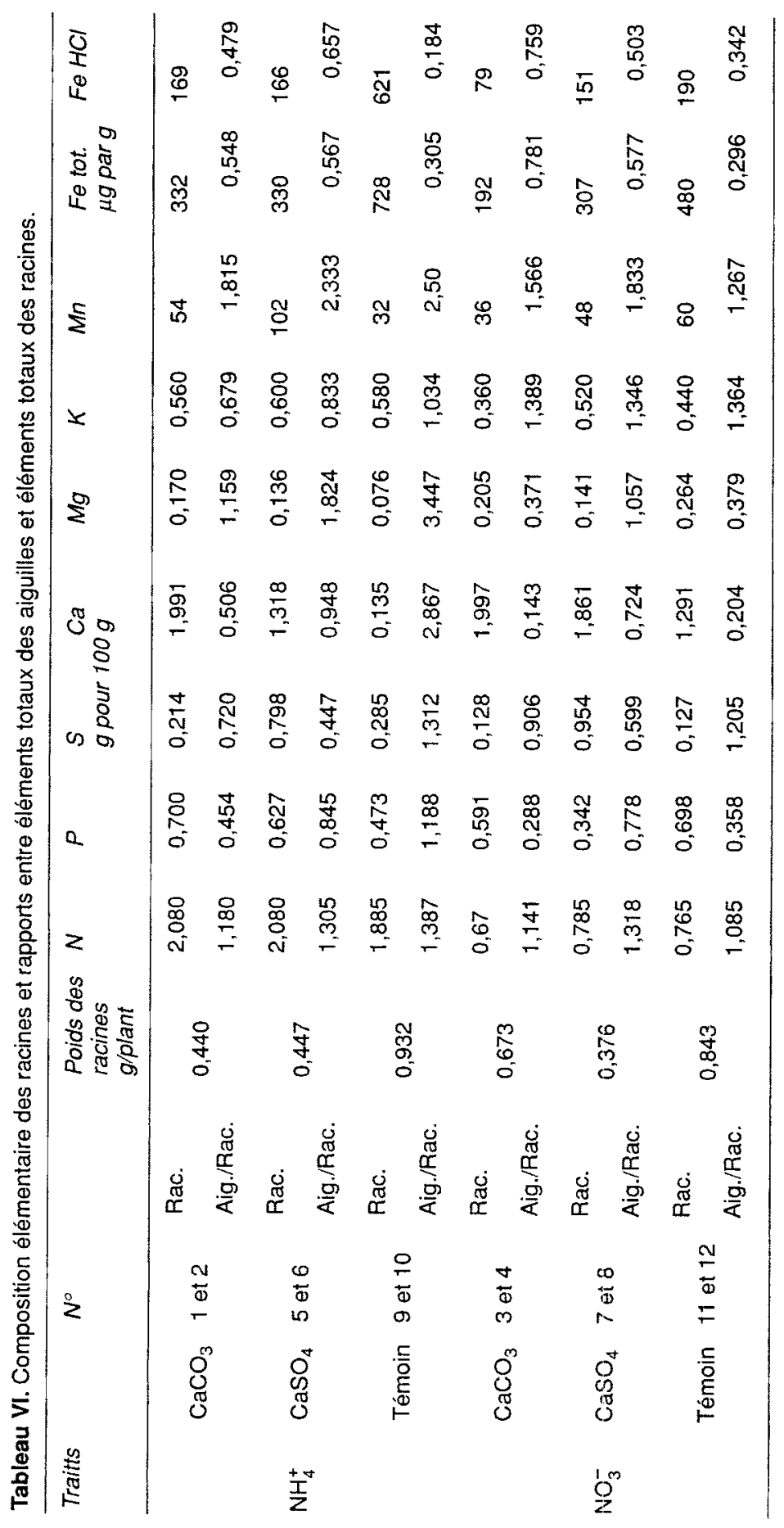


Le calcium parvient en surabondance aux aiguilles et surtout s'accumule fortement au niveau des racines avec précipitation sous forme de carbonate autour des racines.

\section{Sous l'effet d'une alimentation azotée exclusivement nitrique}

En effet, la croissance des parties aériennes est alors nettement diminuée par rapport aux témoins alimentés uniquement en ammonium, mais celle des racines est moins affectée.

La teneur en azote des aiguilles est extrêmement faible : le sapin de Nordmann semble très mal absorber les nitrates. II est d'ailleurs remarquable que la teneur en azote soluble des aiguilles reste très inférieure à celle des témoins recevant de l'azote ammoniacal. On peut, autant et peut-être plus que l'épicéa, le qualifier de "nitratophobe".

La présence de calcium ne déprime pas davantage la croissance et l'alimentation des aiguilles. Le calcium est beaucoup moins absorbé, au niveau foliaire qu'en cas d'alimentation ammoniacale, surtout en présence de calcaire. Mais il n'en est pas de même pour les racines qui s'enrichissent notablement en calcium, même en l'absence de $\mathrm{CaCO}_{3}$.

La teneur en fer "actif» est plus faible qu'en milieu ammoniacal, tout en ne s'abaissant pas à de très faibles valeurs, que ce soit dans le témoin ou en présence de calcaire. La teneur en potassium des aiguilles n'est que peu déprimée. Ce cas de chlorose apparaît donc comme notablement différent de la chlorose calcaire en milieu ammoniacal, sauf au niveau racinaire.
Comment rapprocher ces observations de la chlorose observée en milieu naturel ?

La chlorose, par excès de calcaire en alimentation ammoniacale, semble, à première vue, un cas théorique puisque la nitrification est toujours active en milieu calcaire et on est effectivement tenté de dire que ce que l'on observe sur le terrain se rapproche davantage de la chlorose nitrique; les teneurs en azote, en calcium et en fer "actif» sont du même ordre de grandeur dans l'expérience en serre et sur le terrain (Khalil, 1987; Khalil et al., 1988). En outre, la nutrition potassique reste bonne comme dans la plantation de la région de Carcassonne.

Cependant, la présence de mycorhizes pourrait, sur le terrain, jouer un rôle vis-àvis de la chlorose par l'intermédiaire de son influence sur la nutrition azotée des plantes. Toutefois, nous ne pouvons aborder une telle question car les plants utilisés dans l'expérience décrite ici, provenant de pépinières étaient pourvus de mycorhizes. En revanche, il existe des cas de "non-chlorose» en milieu très calcaire, sur le sapin de Nordmann, mais dans des plantations effectuées sous le couvert d'un taillis préexistant.

S'agit-il de facteurs génétiques, ou bien le fort éclairement des plantations proches de Carcassonne, effectuées en plein découvert, est-il un facteur d'aggravation de la chlorose ? Laatsch (1967) a cité de nombreux cas de «photolabilité» de la chlorophylle en condition d'alimentation minérale déficiente.

La chlorose reste, de toute évidence, un phénomène complexe lié à la co-occurrence de plusieurs facteurs chimiques, physiques et biologiques. 


\section{REMERCIEMENTS}

Notre reconnaissance va à $\mathrm{M}$. Bitsch et $\mathrm{A}$. Clement du CNRF et à E. Jeanroy et J. Rouiller du CPB pour leur contribution aux analyses chimiques; à D. Xeuxet du CNRF pour son aide dans l'analyse statistique et a A. Mariotti du laboratoire de biogéochimie des isotopes stables de l'université $P$. et $M$. Curie de Paris, pour l'analyse des isotopes du carbone.

\section{RÉFÉRENCES}

Anderson C.A. (1982) The effect of high pH and $P$ on the development of lime-chlorosis in two seedling populations of Eucalyptus obliqua L'Hérit. Plant Soil, 69, 199-212

Association Technique pour la Vulgarisation Forestière (1966) Le Sapin de Nordmann. Bull. Vulgarisation Forestière, $66 / 10,13,1-4$

Bachacou J., Masson J.P. \& Millier C. (1981) Manuel de la programmathèque statistique, Amance. Département de Biométrie du CNRS, INRA, $516 \mathrm{p}$.

Brown J.C. (1960) An evaluation of bicarbonate induced chlorosis. Soil Sci. 89, 5

Callot G., Chamayou H., Maertens C. \& Salsac L. (1982) Mieux comprendre les interactions sol-racine. Incidence sur la nutrition minérale. INRA, Versailles Publ., 325 p.

Clement A. (1977) Point 77 sur certaines analyses foliaires. CNRF, Station des Sols Forestiers, Publ. Interne, $24 \mathrm{p}$.

Clement A., Garbaye J. \& Le Tacon F. ( 1977) Importance des ectomycorhizes dans la résistance au calcaire du Pin noir Pinus nigra (Arn. ssp. nigricans Host.). Oecol. Plant. 12, 111-131

Deines P. (1980) The isotopic composition of reduced organic carbon. In : "Handbook of Environmental Isotope Geochemistry" (P. Fritsch and J. Ch. Fontes, Eds.), Vol. 1A, 329406

Emrich K., Ehmalt D.H. \& Vogel P.C. (1970) Carbon isotope fractionation during the precipitation of calcium carbonate. Earth Plan. Sci. Let., 8, 363-371

Etaix G. (1970) Essai de fertilisation sur Abies nordmanniana. Rev. For. Fr., 22, 60-62

Evers F.H. (1964) Die Bedeutung des Stickstofform für das Wachstum und die Ernährung der
Pflanzen insbesondere der Waldbäume. Mitteil. Ver. forstl. Standortskde Forstpflanz., 14, 19-37

Fiedler H.J., Nebe W. \& Hoffmann F. (1973) Forstliche Pflanzenernährung und Düngung. VEB Gustav Fischer, Fence, 481 p.

Frison G., Anselmi N. \& Boccone A. (1982) Research on iron chlorosis of poplars. FAO Intern. Poplar Commission, $57 \mathrm{p}$.

Garbaye J. \& Le Tacon F. (1974) Etude expérimentale du comportement de divers clones de résineux et de deux clones de peupliers vis-àvis du $\mathrm{pH}$ et de la teneur en calcaire du sol. Rev. For. Fr., XXVI, (6), 439-446

Gay-Bellile F. (1972) La chlorose calcaire de la vigne. Proc. Agric. Vitic., 13, 326-332

Gouny P. \& Mazoyer R. (1953) Relation entre la nutrition minérale et les symptômes pathologiques dans la chlorose calcaire. Ann. Agron. 4, 561-598

Jaillard B. (1985) Activité racinaire et rhizostructures en milieu carbonaté. Pédologie, Gand, XXXV, 297-313

Khalil N. (1987) La chlorose calcaire du sapin de Nordmann, Abies nordmanniana Spach (1842) (A. Leioclada Steven). Etude des relations entre l'intensité des symptômes chlorotiques et les propriétés du sol. Influence du type d'alimentation azotée. Thèse de l'Université de Nancy I, Science du Sol-Nutrition Végétale, $142 \mathrm{p}$.

Khalil N., Guillet B. \& Bonneau M. (1988) Comportement du sapin de Nordmann en sol calcaire. Rev. For. Fr.

Laatsch W. (1967) Beziehungen zwischen Standortsfaktoren, Ernährungszustand und Wuchsleistung. XIV Congr. IUFRO, Munich, 1967, II, 22-34

Le Tacon F. (1976) La présence de calcaire dans le sol. Influence sur le comportement de l'épica commun (Picea excelsa Link) et du Pin noir d'Autriche (Pinus nigra nigricans Host.). Thèse de Doctorat d'Etat, INPL-Université de Nancy I, 214 p.

Le Tacon F., Timbal J. \& Valdenaire J.M. (1982) Influence de la forme d'azote minéral sur la croissance d'espèces herbacées forestières. Oecol. Plant., 3, 307-318

Loué A. (1983) Déficiences en oligoéléments actuellement reconnues sur les plantes cultivées en France ( $\mathrm{Fe}, \mathrm{Mn}, \mathrm{Zn}, \mathrm{Cu}, \mathrm{B}, \mathrm{Mo}$ ) au cours de la dernière décennie. Sci. Sol., 2, 89107

Morlat R., Dupont J. \& Salette J. (1980) Aspects écologiques de la manifestation de la 

chlorose ferrique en année sèche chez la vigne dans les sols calcaires de la moyenne vallée de la Loire. Ann. Agron., 31, 219-238

Oserkowsky J. (1933) Quantitative relation between chlorophyll and iron in green and chlorotic pean leaves. Plant Physiol, 8, 449-468

Pourtet J. (1951) Les repleuplements artificiels. Ecole Nationale des Eaux et Forêts, $2^{\circ}$ éd., $242 \mathrm{p}$.

Rehfuess K.E. (1967) Standort und Ernährungszustand von Tannenbeständen (Abies alba Mill.) in der südwestdeutschen
Schichtstufenlandschaft. Fortwiss. Cbl. 86, 321 348

Venkat Rajin K., Marschner H. \& Romheld V. (1972) Effect of iron nutritional status on ion uptake, substrate $\mathrm{pH}$ and production and release of organic acids and riboflavin by sunflower plants. Z. Pflanzenernährung Bodenk., 132, 177-190

Zech W. (1970) Nadelanalytische Untersuchungen über die Kalkchlorose der Waldkiefer (Pinus silvestris). $Z$. Pflanzenernährung Bodenk. 125, 1-16 